\title{
Atomistic Simulations of Formation of Elementary Zr-I Systems
}

\author{
Matthew L. Rossi, Christopher D. Taylor \\ Materials Science and Technology, MST-6, Los Alamos National Lab, Los Alamos, USA \\ E-mail:mrossi@lanl.gov \\ Received August 6, 2011; revised September 8, 2011; accepted October 14, 2011
}

\begin{abstract}
We report results of simulations on the formation of simple zirconium iodide molecules. Previous work by Wimmer et al. [1] explored the relationship between iodine and a zirconium surface. We investigate the reaction schemes through atomistic simulations to better understand the nature of $\mathrm{Zr}$-I interactions through isolated molecules. The computed energy values of varying $\mathrm{Zr}$-I systems suggests a strong binding mechanism between zirconium and iodine, and offer predictions of likely reaction products. The computed results predict condensation of volatile $\mathrm{ZrI}_{4}$ with $\mathrm{ZrI}_{2}$ to form $\mathrm{Zr}_{2} \mathrm{I}_{6}$.
\end{abstract}

Keywords: Nuclear Chemistry, Quantum Chemistry, Computational Modelling

\section{Introduction}

Considerable amounts of research have been performed regarding zirconium and zirconium-based alloys in the past few decades, due to usage in cladding for light water nuclear reactors (LWR).Stress-corrosion cracking (SCC) of the cladding has been observed [2] over the course of fuel-cycle lifetimes in nuclear reactors; the exact initiation of which, however, remains yet unclear [3,4]. Much research has been done regarding iodine-influenced SCC, (I-SCC), which regards iodine as a strong accelerating factor in the corrosion of the zirconium cladding [5-7].

In LWRs, the uranium fuel pellets are enclosed within a zirconium-based alloy, such as Zircaloy-4 or Zirlo. These materials are chosen due to the very low crosssection of zirconium with respect to thermal neutrons. However, these materials can undergo significant corrosion due to stress cracking and fission product reactivity, in addition to other sources such as radiation or hydrogen embrittlement. The effect of iodine, a fission product of uranium, can affect the system in several ways. The first is direct chemical interaction and changes to the basal layer morphology characteristics due to bonding; the second, a physical localized stress induced via iodine adsorption. It is likely that I-SCC is caused by a combination of both effects on the exposed $\mathrm{Zr}$ surface, and investigation is required to better understand the initiation and nucleation mechanics of I-SCC. Physical stress on the system can be caused by gas pressure of the fis- sion products on the cladding walls. The internal fuel rod pressures can range from $1-14 \mathrm{MPa}$ during standard operating conditions, up to $1 \mathrm{GPa}$ in contained "bubbles", [8] with pressure increasing [9] with burn-up. The focus of this research is the chemical interactions between zirconium and iodine.

The $\mathrm{Zr}$ cladding is expected to react strongly with the released iodine during fission; iodine is indeed used to purify $\mathrm{Zr}$ metal in a filament method [10]. Under this scheme, iodine is used such that:

$$
\begin{gathered}
\mathrm{Zr}+2 \mathrm{I}_{2} \leftrightarrow \mathrm{ZrI}_{4} \\
2 \mathrm{I} \leftrightarrow \mathrm{I}_{2}
\end{gathered}
$$

with the combination of the impure $\mathrm{Zr}$ and $\mathrm{I}_{2}$ gas forming volatile $\mathrm{ZrI}_{4}$, which then deposits on a filament, and can decompose under the equilibrium of (1), resulting in pure zirconium. This same mechanism is suspected to be a means of I-SCC in Zr cladding [11]. Formation of zirconium iodides, however, can occur in multiple stoichiometries. There are structures provided in the Inorganic Crystal Structure Database (ICSD) for $\mathrm{ZrI}_{2}$ [12], $\mathrm{ZrI}_{3}$ [13], in addition to $\mathrm{ZrI}_{4}$ [14]. One must consider each of these molecular crystals in the effort to understand I-SCC, as it is likely that as iodine gas evolves from the fuel pellet, the surface concentration of iodine increases, and the surface $\mathrm{Zr}$ atoms undergo changes in crystal morphology to reflect the current $\mathrm{ZrI}_{\mathrm{x}}$ stoichiometry. Shown in Equation (2), evolution of free iodine atoms occurs during fission, which can combine to form $\mathrm{I}_{2}$ gas, in cooperative equilibrium with (1). 
The formation of zirconium iodides are important due to observations seen in previous research [15-17], which can lead to pit formation and nucleation $[5,11]$, followed by material failures. A suspected mechanism of pit formation is due to weakening of $\mathrm{Zr}-\mathrm{Zr}$ bonds [7] at and around the site of iodine adsorption. Once these bonds are sufficiently weakened, it is likely that the zirconium surface undergoes a period of iodine aggregation, where the material proceeds from $\mathrm{Zr}_{\mathrm{x}} \mathrm{I}_{1-\mathrm{x}}$ to a locally saturated state approaching stoichiometric $\mathrm{ZrI}_{4}$. While this sort of mechanism may not describe the bulk surface, such aggregation is indeed likely to occur at or near grain boundaries, where $\mathrm{Zr}-\mathrm{Zr}$ bonding is significantly weaker than along a pristine surface.

The research contained within this paper investigates the agglomeration of iodine on zirconium atoms in isolated systems.

\section{Methodology}

In an effort to understand the mechanistics of Zr-I interactions, simple $\mathrm{ZrI}_{\mathrm{x}}$ molecular species were constructed and energy structure calculations were performed. These molecular species were chosen due to their corresponding stoichiometries as given by the ICSD structures [12-14]. In addition, mapping the potential energy of dissociation for $\mathrm{Zr}_{2} \mathrm{I}_{6}$ was performed. $\mathrm{ZrI}_{4}$ volatilizes at relatively low temperature [7] and can evolve from an iodine-saturated $\mathrm{Zr}$ surface during reactor operation. Therefore, understanding the iodine aggregation on the $\mathrm{Zr}$ surface can provide insight to pitting and cracking [11] which occur during cladding failure.

It is notable that $\mathrm{Zr}$-I species undergo changes to spin state due to bonding. $\mathrm{ZrI}_{2}$ is expected to exist in the singlet state, while $\mathrm{ZrI}_{3}$ is in the doublet state and $\mathrm{ZrI}_{4}$ is a singlet. The dimerized system, $\mathrm{Zr}_{2} \mathrm{I}_{6}$, is once again a singlet. This can introduce problematic convergence in systems due to crossover from triplet-to-singlet states as the isolated materials approach. The reaction schemes are as follows (shown in Figure 1):

$$
\begin{gathered}
\mathrm{ZrI}_{2}+\mathrm{ZrI}_{4} \leftrightarrow \mathrm{Zr}_{2} \mathrm{I}_{6} \\
2 \mathrm{ZrI}_{3} \leftrightarrow \mathrm{Zr}_{2} \mathrm{I}_{6}
\end{gathered}
$$

Note that in $(3 b)$, the reactants are in the triplet state; while this triplet state is caused by the presence of two doublets, a singlet state is also possible. The reverse reaction of $3 \mathrm{a}$ and $3 \mathrm{~b}$ are disproportionation (dis) and comproportionation (com), respectively.

Additional reaction schemes were investigated to study the propagation of iodizing zirconium. These schemes were used to simulate the means by which $\mathrm{Zr}$ metal results in the volatile $\mathrm{ZrI}_{4}$ species, which has been observed to cause pitting and corrosion $[5,11]$. Elemen-

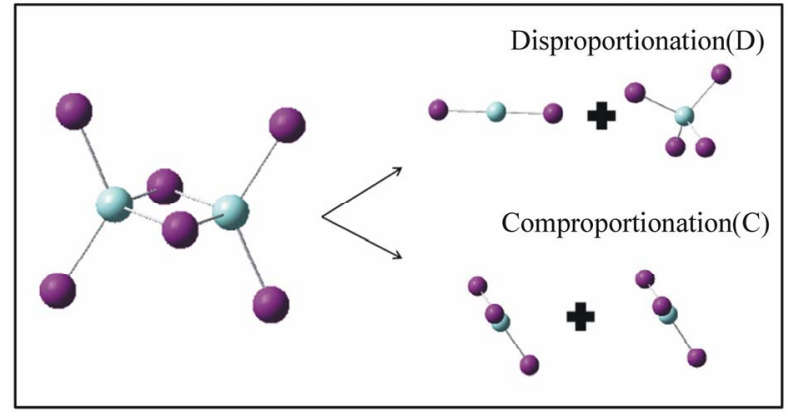

Figure 1: Reaction schematic for the decomposition of $Z_{r_{2}} I_{6}$ into disproportionation (upper) and comproportionation (lower) components. Electronic structure calculations predict formation of $\mathrm{Zr}_{2} \mathrm{I}_{6}$ from both the $\mathrm{D}$ and $\mathrm{C}$ materials.

tary formations of higher-valency iodides were formed under the following reactions:

$$
\begin{gathered}
\mathrm{Zr}+\mathrm{I}_{2} \leftrightarrow \mathrm{ZrI}_{2} \\
\mathrm{ZrI}_{2}+\mathrm{I}_{2} \leftrightarrow \mathrm{ZrI}_{4} \\
2 \mathrm{ZrI}_{2}+\mathrm{I}_{2} \leftrightarrow 2 \mathrm{ZrI}_{3} \\
2 \mathrm{ZrI}_{3}+\mathrm{I}_{2} \leftrightarrow 2 \mathrm{ZrI}_{4}
\end{gathered}
$$

All calculations were performed with the GAMESS $[18,19]$ software package using the hybrid B3LYP [20-22] functional with the SBKJC [23-25] basis set.

\section{Results and Discussion}

$\mathrm{ZrI}_{2}$ was calculated in both the singlet and triplet state, based on the ground state electron configuration of $\mathrm{Zr}$ $\left({ }^{3} \mathrm{~F}_{2}\right)$. In the case of $\mathrm{ZrI}_{2}$, the singlet state is preferred by a value of $1.24 \mathrm{eV}$. This is consistent with bonding to the d-orbital and the presence of a non-bonding fully occupied Zr s-orbital.

By computation of (3a), we observe that the equilibrium favors the product, $\mathrm{Zr}_{2} \mathrm{I}_{6}$, and does not undergo disproportionation. The energy change of the forward reaction (3a) is $3.32 \mathrm{eV}$. The reaction pathway between the products and reactants in $(3 \mathrm{~b})$ is also exothermic, with computed value of $1.79 \mathrm{eV}$. Therefore, in the event of dissociation, the overwhelmingly favored product would be comproportionation to $\mathrm{ZrI}_{3}$, with an energetic favoring of $1.54 \mathrm{eV}$ relative to the disproportionation products.

Formation of $\mathrm{Zr}_{2} \mathrm{I}_{6}$ from component materials, as shown in $(3 a, b)$, is of primary interest. As originally reported by Busol [26], there are two possible mechanisms in $\mathrm{ZrI}_{\mathrm{x}}$ formation:

$$
\begin{array}{r}
3 \mathrm{ZrI}_{4}+\mathrm{Zr} \leftrightarrow 4 \mathrm{ZrI}_{3} \\
\mathrm{ZrI}_{4}+\mathrm{Zr} \leftrightarrow 2 \mathrm{ZrI}_{2}
\end{array}
$$

Busol [26] determined that the rate limited step in $\mathrm{ZrI}_{3}$ formation was not $\mathrm{I}_{2}$ concentration, but rather, the diffu- 
sion rate at which $\mathrm{I}_{2}$ can pass through surface $\mathrm{ZrI}_{\mathrm{x}}$ layers to reach pristine $\mathrm{Zr}$. Observations from their work have shown that formation of $\mathrm{ZrI}_{3}$ is the primary reaction, with little or no formation of $\mathrm{ZrI}_{2}$. This is consistent with the energetic barrier we observe to the formation of $\mathrm{ZrI}_{2}$ from $\mathrm{Zr}_{2} \mathrm{I}_{6}$, which at high temperature the latter can decompose. This, however, does not preclude volatilization of $\mathrm{ZrI}_{4}$, which can form when the exposed $\mathrm{Zr}$ surface is sufficiently saturated with iodine, such that the disproportionation reaction (Equation (3a)) need not occur to yield $\mathrm{ZrI}_{4}$.

In a system containing a pair of $\mathrm{ZrI}_{3}$ molecules, separated at $6 \AA$ beyond the equilibrium separation of $\mathrm{Zr}$ atoms in $\mathrm{Zr}_{2} \mathrm{I}_{6}$ (from here on, denoted eq $+6 \AA$ ), the preferred electronic state is to remain as two doublets which combine for an overall triplet state, energetically favored by $1.43 \mathrm{eV}$ relative to that of the singlet state. This energy difference is evidence of errors within Hartree-Fock (HF) methods, as two isolated $\mathrm{ZrI}_{3}$ molecules should exhibit identical energies regardless of being in the singlet or triplet state. Upon inspection of the atomic charges, it does not appear that any ionization of species occurred, but that the $\mathrm{ZrI}_{3}$ molecules were essentially symmetric in charge distribution. Examination of the molecular orbitals (Figures 2-3) show the loss of degeneracy in the isolated $\mathrm{ZrI}_{3}-\mathrm{ZrI}_{3}$ singlet state, as is expected. However, the population of the non-degenerate HOMO shows electron density primarily in the d-orbitals, suggesting a delocalized, higher energy orbital occupation which does not occur when the system is allowed to exist in the triplet state, as a sum of two doublets.

Higher level theory was implemented (MCSCF [27-31]/SBKJC) to investigate this deviation, with resultant energy between the singlet and triplet states showing negligible $\left(4.55 \times 10^{-5} \mathrm{eV}\right)$ difference. Regardless of choosing restricted or restricted open-shell Hartree-Fock, HF-based methods fail to properly describe two isolated doublets, as these methods do not properly handle the multi-configurational spin states involved. Therefore, the energy difference predicted between singlet and triplet isolated $\mathrm{ZrI}_{3}$ pairs is an artifact of Hartree-Fock theory. However, MCSCF is significantly more computationally expensive than DFT, and therefore is useful for the investigation of the difference between singlet and triplet states, but impractical for the high volume of calculations required to determine the potential energy curve mapped in Figure 4. The energy of the dissociated ((eq) $+6 \AA)$ disproportionation products was also computed to make a comparison between the dis and com lowest-energy products relative to the DFT results shown in Figure 4, with an energy difference of $2.05 \mathrm{eV}$ favoring the comproportionation products. This energy is slightly larger than DFT-predicted result of $1.54 \mathrm{eV}$, both favoring

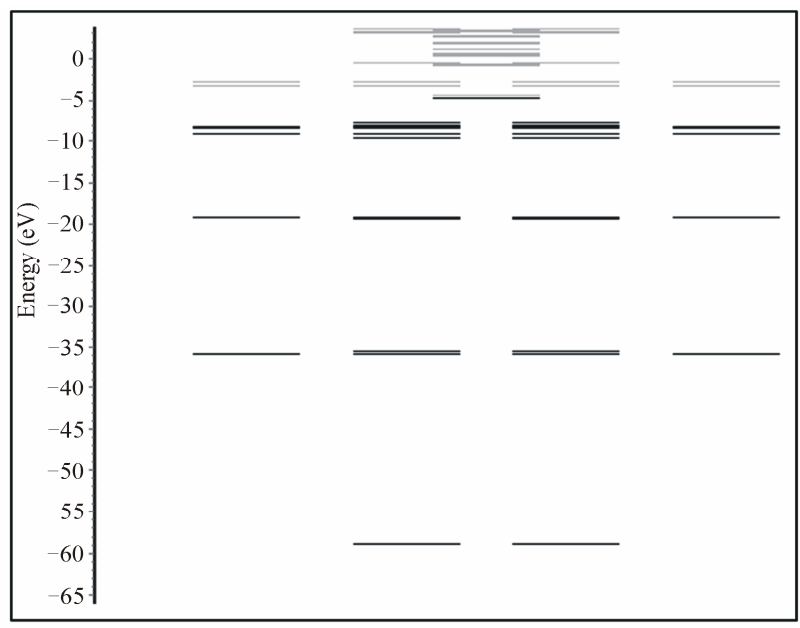

Figure 2. Molecular orbital diagram of $\mathrm{ZrI}_{3}-\mathrm{ZrI}_{3}$ singlet system at eq $+6.0 \AA$ separation. Black indicates occupied orbitals, grey indicates virtual (unoccupied) orbitals.

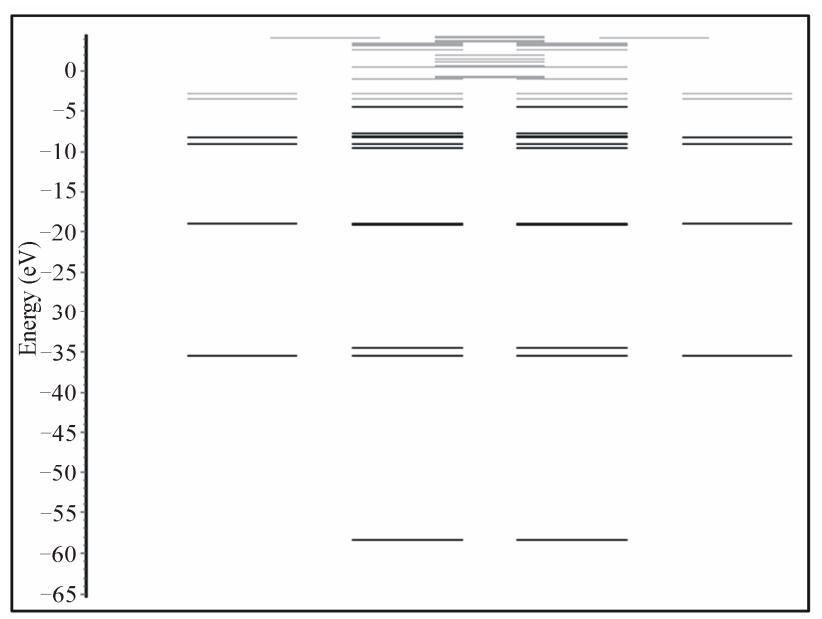

Figure 3. Molecular orbital diagram of $\mathrm{ZrI}_{3}-\mathrm{ZrI}_{3}$ triplet system at eq $+6.0 \AA$ separation. Black indicates occupied orbitals, grey indicates virtual (unoccupied) orbitals.

formation of $2 \mathrm{ZrI}_{3}$ in the dissociation of $\mathrm{Zr}_{2} \mathrm{I}_{6}$.

Mapping of the potential energy curve for the reaction coordinate (Figure 4) for combination/dissociation of $\mathrm{Zr}_{2} \mathrm{I}_{6}$ to the comproportionation and disproportionation products shows that at approximately Zr-equilibrium (eq) $+1 \AA$ separation, the $\mathrm{Zr}_{2} \mathrm{I}_{6}$ system undergoes a spin transformation from the singlet $\mathrm{Zr}_{2} \mathrm{I}_{6}$ to a roughly associated triplet $\mathrm{ZrI}_{3}-\mathrm{ZrI}_{3}$ pair.

The combination schemes (3a, 3b, shown in Figure 4) reveal that in the region between the equilibrium $\mathrm{Zr}-\mathrm{Zr}$ separation of $Z_{2} I_{6}$ to a separation of $\sim 2 \AA$, both the dis and com behave identically, reaching a stable, loosely bound $\mathrm{Zr}_{2} \mathrm{I}_{6}$ when the iodines are allowed to relax during a constrained $\mathrm{Zr}-\mathrm{Zr}$ distance optimization. However, as the separation increases, the asymptotic limit is reached 


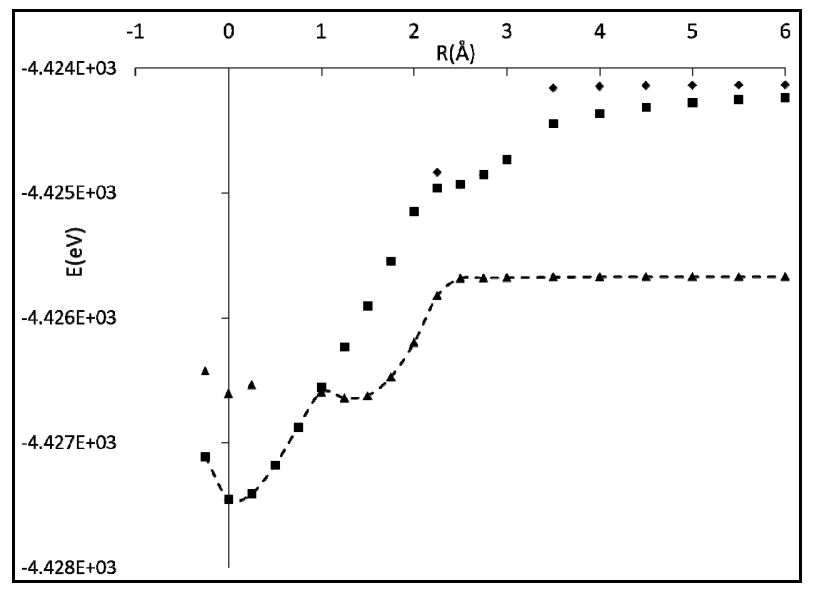

Figure 4. Energy curves for disproportionation (diamonds) and comproportionation in the singlet (squares) and triplet (triangles) states relative to displacement from the equilibrium position. The dashed line indicates the suspected path which includes conversion from the isolated triplet (two doublet) state of $\mathrm{ZrI}_{3}$ to combination resulting in the singlet $\mathbf{Z r}_{2} \mathbf{I}_{6}$.

for the dis and com products, respectively, which differ slightly in energy. It can be noted, however, that due to the preference for the singlet-triplet transition of the com products at a separation of $\sim$ eq $+1 \AA$, the com reaction will instead follow the dashed (low) path (Figure 4).

The reactions show in Equations (4)-(5) yielded exothermic results, consistent with increasing iodine coordination to the $\mathrm{Zr}$ central atoms. The associated energies of these reactions is shown in Table 1. The computed enthalpies of Equations (4b) and (5a) are lower the energy change associated with $5 \mathrm{~b}$. The data suggests that there is a preference to the formation of $\mathrm{ZrI}_{3}$, rather than $\mathrm{ZrI}_{4}$, again, supported by the report by Busol [26]

\section{Conclusions}

It is apparent that aggregation of $\mathrm{ZrI}_{\mathrm{x}}$ species will form larger systems as shown in structures from the ICSD [12-14]. The choice of studying $\mathrm{Zr}_{2} \mathrm{I}_{6}$ was based on the analogous structure of bulk $\mathrm{ZrI}_{2}$ [12], which is not stoichiometrically identical, but geometrically similar as the system size increases. (The excess of iodine in the stoichiometry is due to terminal iodine capping.) For example, the next largest structure, $\mathrm{Zr}_{3} \mathrm{I}_{8}$, with each $\mathrm{Zr}$ atom having two bridging iodines, and maintaining a tetrahedral geometry of the $\mathrm{Zr}$ cores, consistent with the bulk $\mathrm{ZrI}_{2}$ structure. Such aggregation supports the idea that as iodine exposure increases, similar to what occurs within a nuclear reactor, the iodine will bind to the $\mathrm{Zr}$ cladding, causing changes to the surface morphology of the cladding material, and enabling the growth of poly crystalline $\mathrm{ZrI}_{2}$. It is expected that upon reaching some
Table 1. Energy change table for reactions 4-5.

\begin{tabular}{cccc}
\hline Reaction & Reactants & Products & $\Delta \mathrm{E}(\mathrm{eV})$ \\
\hline $4 \mathrm{a}$ & $\mathrm{Zr}+\mathrm{I}_{2}$ & $\mathrm{ZrI}_{2}$ & -4.59 \\
$4 \mathrm{~b}$ & $\mathrm{ZrI}_{2}+\mathrm{I}_{2}$ & $\mathrm{ZrI}_{4}$ & -6.78 \\
$5 \mathrm{a}$ & $2 \mathrm{ZrI}_{2}+\mathrm{I}_{2}$ & $2 \mathrm{ZrI}_{3}$ & -9.50 \\
$5 \mathrm{~b}$ & $2 \mathrm{ZrI}_{3}+\mathrm{I}_{2}$ & $2 \mathrm{ZrI}_{4}$ & -5.24 \\
\hline
\end{tabular}

critical limit, the surface iodine saturation will cause morphological changes to the $\mathrm{Zr}$ surface, resulting in a mixture of $\mathrm{ZrI}_{\mathrm{x}}$ species, and ultimately to stoichiometric $\mathrm{ZrI}_{4}$. Upon reaching iodine surface saturation, it is then possible that molecular $\mathrm{ZrI}_{4}(\mathrm{~g})$ volatilization will be possible, resulting in pitting, and ultimately, corrosion of the cladding.

\section{Acknowledgements}

This research was supported by the Consortium for Advanced Simulation of Light Water Reactors (www. casl.gov), an Energy Innovation Hub (http://www.energy.gov/hubs) for Modeling and Simulation of Nuclear Reactors under U.S. Department of Energy Contract No. DE-AC05-00OR22725. The Los Alamos National Laboratory is operated by Los Alamos National Security LLC for the National Nuclear Security Administration of the U.S. Department of Energy under contract DE-AC5206NA25396.

\section{References}

[1] E. Wimmer, R. Najafabadi, et al., “Ab Initio Calculations for Industrial Materials Engineering: Successes and Challenges," Journal of Physics: Condensed Matter, Vol. 22, No. 38,2010 , p. 384215. doi:10.1088/0953-8984/22/38/384215

[2] S. A. Nikulin and A. B. Rozhnov, "Corrosion Cracking of Zirconium Cladding Tubes (A Review). I. Methods of Study and Mechanisms of Fracture," Metal Science and Heat Treatment, Vol. 47, No. 1-2, 2005, pp. 71-79. doi:10.1007/s11041-005-0034-2

[3] S. B. Goryachev, A. R. Gritsuk, et al., "Iodine Induced SCC of Zr Alloys at Constant Strain Rate," Journal of Nuclear Materials, Vol. 199, No. 1, 1992, pp. 50-60. doi:10.1016/0022-3115(92)90439-R

[4] V. V. Likhanskii and L. V. Matweev, "The Development of the Crack Growth Model in Zirconium Claddings in Iodine Environment," Nuclear Engineering and Design, Vol. 213, No. 2-3, 2002. pp. 133-140. doi:10.1016/S0029-5493(01)00516-7

[5] A. Serres, L. Fournier, et al., "The Effect of Iodine Content and Specimen Orientation on Stress Corrosion Crack Growth Rate in Zircaloy-4," Corrosion Science, Vol. 52, No. 6, 2010, pp. 2001-2009. doi:10.1016/j.corsci.2010.02.008 
[6] I. Schuster and C. Lemaignan, "Influence of Texture on Iodine-Induced Stress Corrosion Cracking of Zircaloy-4 Cladding Tubes," Journal of Nuclear Materials, Vol. 189, No. 2, 1992, pp. 157-166. doi:10.1016/0022-3115(92)90528-S

[7] P. P. S. Sidky, "Iodine Stress Corrosion Cracking of Zircaloy Reactor Cladding: Iodine Chemistry (a Review)," Journal of Nuclear Materials, Vol. 256, No. 1, 1998, pp. 1-17. doi:10.1016/S0022-3115(98)00044-0

[8] F. Lemoine, "High Burnup Fuel Behavior Related to Fission Gas Effects under Reactivity Initiated Accidents (RIA) Conditions," Journal of Nuclear Materials, Vol. 248, No. 1, 1997, pp. 238-248. doi:10.1016/S0022-3115(97)00157-8

[9] P. P. Rudling, R. Adamson, et al., "High Burnup Fuel Issues," Nuclear Engineering Technology, Vol. 40, 2008, $1-8$.

[10] K. D. Sinel'nikov, F. I. Busol and G. I. Stepanova, "Problem of the Iodine Method of Purification of Zirconium," Atomic Energy, Vol. 4, 1958. pp. 221-227.

[11] S. Y. Park, et al., "Crack Initiation and Propagation Behavior of Zirconium Cladding under an Environment of Iodine-Induced Stress Corrosion," Metals and Materials International, Vol. 13, No. 2, 2007, pp. 155-163. doi:10.1007/BF03027567

[12] D. H. Guthrie and J. D. Corbett, "Synthesis and Structure of an Infinite-Chain Form of Zr I2 (alpha)," JSSC, Vol. 37, 1981, pp. 256-263.

[13] A. Lachgar, D. S. Dudis and J. D. Corbett, "Revision of the Structure of Zirconium Triiodide. The Presence of Metal Dimers," Inorganic Chemistry, Vol. 29, No. 12, 1990, pp. 2242-2246. doi:10.1021/ic00337a013

[14] B. Krebs, G. Henkel and M. Dartmann, "Kristallstruktur von Zirkoniumtetrajodid $\mathrm{Zr}$ I4. Ein Neuer A B4Strukturtypp," Acta Crystallographica Section B, Vol. 35, 1979, pp. 274-278. doi:10.1107/S0567740879003344

[15] S. B. Farina, G. S. Duffo and J. R. Galvele, "Localized Corrosion of Zirconium and Zircaloy-4 in Iodine Alcoholic Solutions," LAAR, Vol. 32, 2002, pp. 295-298.

[16] S. B. Farina, G. S. Duffo and J. R. Galvele, "Stress Corrosion Cracking of Zircaloy-4 in Halide Solutions. Effect of Temperature," Materials Research, Vol. 5, No. 2, 2002, pp. 107-112. doi:10.1590/S1516-14392002000200004

[17] P. P. Jacques, F. Lefebvre and C. Lemaignan, "Deformation-Corrosion Interactions for $\mathrm{Zr}$ Alloys during ISCC Crack Initiation. Part I: Chemical Contributions," Journal of Nuclear Materials, Vol. 264, No. 3, 1999, pp. 239-248. doi:10.1016/S0022-3115(98)00501-7

[18] M. W. Schmidt, K. K. Baldridge, et al., "General Atomic and Molecular Electronic Structure System," Journal of Computational Chemistry, Vol. 14, No. 11, 1993, pp. 1347-1363. doi:10.1002/jcc.540141112

[19] M. S. Gordon and M. W. Schmidt, "Advances in Elec- tronic Structure Theory: GAMESS a Decade
Later," In: C. E. Dykstra, G. Frenking, et al., Eds., Theory and App- lications of Computational Chemistry, the First Forty Years, Elsevier, Amsterdam, 2005, pp. 1347-1363. doi:10.1016/B978-044451719-7/50084-6

[20] A. D. Becke, "Density-Functional Thermochemistry. III. The Role of Exact Exchange," Journal of Chemical Physics, Vol. 98, 1993, pp. 5648-5642. doi: $10.1063 / 1.464913$

[21] P. P. J. Stephens, F. J. Devlin, et al., "Ab Initio Calculation of Vibrational Absorption and Circular Dichroism Spectra Using Density Functional Force Fields," The Journal of Physical Chemistry, Vol. 98, No. 45, 1994, pp. 11623- 11627. doi:10.1021/j100096a001

[22] R. H. Hertwig and W. Koch, "On the Parameterization of the Local Correlation Functional. What Is Becke3-LYP?" Chemical Physics Letters, Vol. 268, No. 5-6, 1997, pp. 345-351. doi:10.1016/S0009-2614(97)00207-8

[23] W. J. Stevens, H. Basch and M. Krauss, "Compact Effective Potentials and Efficient Shared-Exponent Basis Sets for the First- and Second-Row Atoms," Journal of Chemical Physics, Vol. 81, No. 12, 1984, pp. 6026-6033. doi:10.1063/1.447604

[24] W. J. Stevens, M. Krauss, H. Basch and P. P. G. Jasien, "Relavtivistic Compact Effective Potentials and Efficient, Shared-Exponent Basis-Sets for the 3rd-Row, 4th-Row and 5th-Row Atoms," Canadian Journal of Chemistry, Vol. 70, No .2, 1992, pp. 612-630. doi:10.1139/v92-085

[25] T. R. Cundari and W. J. Stevens, "Effective Core Potential Methods for the Lanthanides," Journal of Chemical Physics, Vol. 98, No. 7, 1993, pp. 5555-5565. doi:10.1063/1.464902

[26] F. I. Busol, "Kinetics of the Reduction of $\mathrm{ZrI}_{4}$ Vapors by Metallic Zirconium," Russian Journal of Physical Chemistry, Vol. 33, 1959, pp. 799-807.

[27] M. W. Schmidt and M. S. Gordon, "The Construction and Interpretation of MCSCF Wavefunctions," Annual Review of Physical Chemistry, Vol. 29, 1998, pp. 233266. doi:10.1146/annurev.physchem.49.1.233

[28] B. O. Roos, "The Multiconfiguration SCF Method," In: S. W. Diercksen, Ed., Methods in Computational Molecular Physics, D. Reidel, Dordrecht, 1983, pp. 161-187.

[29] J. Olsen, D. L. Yeager and P. P. Jorgensen, "Optimization and Characterization of a MCSCF State," Advances in Chemical Physics, Vol. 54, 1983, pp. 1-176. doi:10.1002/9780470142783.ch1

[30] H. -J. Werner, "Matrix Formulated Direct MCSCF and Multiconfiguration Reference CI Methods," Advances in Chemical Physics, Vol. 69, 1987, pp. 1-62. doi:10.1002/9780470142943.ch1

[31] R. Shepard, "The MCSCF Method," Advances in Chemical Physics, Vol. 69, 1987, pp. 63-200. doi:10.1002/9780470142943.ch2 